\begin{tabular}{|c|l|}
\hline Title & A coustic interference in random superlattices \\
\hline Author(s) & Tamura, Shin-ichiro; Nori, Franco \\
\hline Citation & $\begin{array}{l}\text { Physical Review B, 41(11), 7941-7944 } \\
\text { https://doi.org/10.1103/PhysRevB.41.7941 }\end{array}$ \\
\hline Issue Date & 1990-04-15 \\
\hline Doc URL & http://hdl.handle.net/2115/5944 \\
\hline Rights & Copyright $\odot$ 1990 A merican Physical Society \\
\hline Type & article \\
\hline File Information & PRB41-11.pdf \\
\hline
\end{tabular}

Instructions for use 


\title{
Acoustic interference in random superlattices
}

\author{
Shin-ichiro Tamura \\ Department of Engineering Science, Hokkaido University, Sapporo 060, Japan
}

Franco Nori*

Physics Department, The University of Michigan, Ann Arbor, Michigan 48109-1120

and Institute for Theoretical Physics, University of California, Santa Barbara, California 93106

(Received 18 January 1990)

\begin{abstract}
We study theoretically the interference effects and the resulting transmission of acoustic phonons in random superlattices, i.e., multilayered systems in which two kinds of building blocks $A$ and $B$ are stacked at random. We derive (i) the recursion relations satisfied by the superlattice structure factors, and (ii) an analytic expression for the ensemble-averaged intensity of phonons reflected from layer interfaces of an infinite system. We predict a number of sharp features in the intensity versus frequency, which appear rather regularly. Furthermore, numerical calculations for both the double- and single-layer random superlattices indeed exhibit distinctive dip structures in phonon transmission spectra which are in good agreement with the analytical results. We also find that the single-layer random superlattices show an effective phonon-filtering action as the system size is increased, i.e., they prevent the transmission of phonons except for those satisfying resonance conditions.
\end{abstract}

Recently, a number of studies ${ }^{1}$ have been devoted to the electronic, vibrational, and structural properties of nonperiodic multilayered systems, or superlattices (SL's). The primary motivation is to understand their physical properties, expected to be quite unlike those of periodic systems. The experimental realization ${ }^{2,3}$ of such systems makes these studies quite appealing.

So far, acoustic-phonon propagation in periodic, quasiperiodic (Fibonacci), and other nonperiodic (ThueMorse) SL's have been studied both theoretically and experimentally. In particular, sharp dips in phonon transmission (which are attributed to both Bragg and Bragg-like reflection processes) are predicted theoretically and observed experimentally in both periodic ${ }^{4}$ and $\mathrm{Fi}$ bonacci ${ }^{5}$ SL's. In the Thue-Morse SL's, ${ }^{6}$ large transmission dips characteristic of the nonperiodicity of the sequence are also predicted in addition to those due to the Bragg reflection.

In this Rapid Communication, we discuss novel interference effects exhibited by high-frequency acoustic phonons in randomly stacked SL's. We obtain these results by deriving (i) the recursion relations satisfied by the SL structure factor, and (ii) an analytic expression for the ensemble-averaged intensity describing the interference effects of phonons reflected from the layer interfaces. In particular, with these expressions, we show that even in random SL's the sharp features are still present in the transmission spectra of phonons and they become more and more significant as the system size is increased. We also show that a certain kind of random SL's have a very effective phonon-filtering action.

Here we consider SL's in which the building blocks $A$ and $B$ consist of double layers as shown in Fig. 1 (a). We further assume that the materials constituting the first (second) layers in these blocks are the same but of different thicknesses in general. In these types of SL's (which we refer to as double-layer SL's), the two kinds of constituent materials appear alternatively even though the sequence of $A$ and $B$ blocks is random. Single-layer SL's in which both $A$ and $B$ blocks consist of single layers [Fig. 1 (b)] are obtained as a limiting case of double-layer SL's, where the thickness of one of the layers in each block tends to zero.

In typical SL's, the acoustic mismatch between the constituent layers is very small and hence the amplitude reflection coefficient $r_{21}\left(=-r_{12}\right)$ of phonons (impinging on an interface from the second layer to the first layer) is also small. [For a (001)-AlAs/GaAs interface, $\left|r_{\text {GaAs,AlAs }}\right|^{2}<0.01$ for both transverse acoustic (TA) and longitudinal acoustic (LA) modes. $\left.{ }^{7}\right]$ Thus, neglecting the multiple reflection of phonons at layer interfaces, the structure factor $S$, in a double-layer SL, satisfies the (a)

A

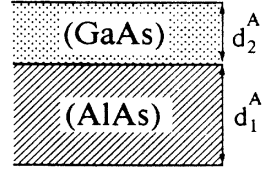

B

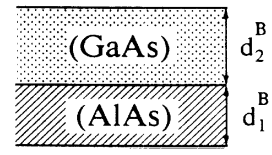

(b)

A

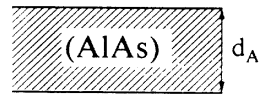

B

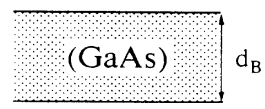

FIG. 1. Schematic representation of the building blocks $A$ and $B$ for (a) double-layer SL's and (b) single-layer SL's. AlAs and $\mathrm{GaAs}$ are assumed to be the constituents of layers in the numerical calculations. 
following recursion relations for the normal propagation

$$
\begin{aligned}
& S_{N}=S_{N-1}+r_{21}\left(1-e^{i \gamma_{N}}\right) \exp \left(i \sum_{l=1}^{N-1} \Theta_{l}\right), N \geq 2, \\
& S_{1}=r_{21}\left(1-e^{i \gamma_{1}}\right),
\end{aligned}
$$

where $N$ is the number of blocks, $\gamma_{N}=2 k_{1} d_{1}^{A} \equiv \alpha_{1}$, and $\Theta_{N}=2\left(k_{1} d_{1}^{A}+k_{2} d_{2}^{A}\right) \equiv a$ (if the $N$ th block is $A$ ) or $\gamma_{N}=2 k_{1} d_{1}^{B} \equiv \beta_{1}$ and $\Theta_{N}=2\left(k_{1} d_{1}^{B}+k_{2} d_{2}^{B}\right) \equiv b$ (if the $N$ th block is $B$ ). In these expressions, $k_{1}$ and $k_{2}$ are the wave numbers of phonons in the first and second layers, respectively.

Now, the intensity of the reflected phonons is described by $\left|S_{N}\right|^{2}$, so we introduce $I_{N}$ defined by

$$
\begin{aligned}
I_{N} \equiv\left|S_{N} / r_{21}\right|^{2} & \\
=I_{N-1}+\left|1-e^{i \gamma_{N}}\right|^{2}+ & {\left[\left(1-e^{-i \gamma_{N}}\right) \exp \left(-i \sum_{l=1}^{N-1} \Theta_{l}\right)\right.} \\
& \left.\times S_{N-1} / r_{21}+\text { c.c. }\right], N \geq 2, \\
I_{1}=\left|1-e^{i \gamma_{1}}\right|^{2} . &
\end{aligned}
$$

The relevant quantity in a random system is the ensemble-averaged intensity $\left\langle I_{N}\right\rangle$ rather than $I_{N}$. In order to calculate $\left\langle I_{N}\right\rangle$, we assume zero short-range correlations in the sequence of blocks and define the following ensemble-averaged quantities $(l=1,2, \ldots, N)$ :

$$
\left\langle e^{-i \Theta_{l}}\right\rangle=\epsilon,\left\langle e^{-i \gamma_{l}}\right\rangle=\eta,\left\langle e^{-i\left(\Theta_{l}-\gamma_{l}\right)}\right\rangle=\xi .
$$

Hence, from Eq. (2), we obtain the recursion relations

$$
\begin{gathered}
\left\langle I_{N}\right\rangle=\left\langle I_{N-1}\right\rangle+\left[(1-\eta)\left(1+(\epsilon-\xi) \frac{1-\epsilon^{N-1}}{1-\epsilon}\right)\right. \\
\quad+\text { c.c. }], N \geq 2, \\
\left\langle I_{1}\right\rangle=2-\eta-\eta^{*} .
\end{gathered}
$$

Solving these equations, we find that for $|\epsilon|<1$ and for a large $N,\left\langle I_{N}\right\rangle$ grows in proportion to $N$, in general. Accordingly, we can derive the expression for the normalized reflected-phonon intensity, $\bar{I}$, in the limit of large $N$,

$$
\bar{I}=\lim _{N \rightarrow \infty}\left\langle I_{N}\right\rangle / N=\frac{(1-\eta)(1-\xi)}{1-\epsilon}+\text { c.c. }
$$

Equation (5) suggests that at frequencies satisfying $\epsilon=1$, $\left\langle I_{N}\right\rangle$ grows as $O\left(N^{2}\right)$ with the system size, unless $\eta=1$ (or $\xi=1)$ is satisfied simultaneously. This corresponds to a Bragg peak in periodic SL's. Whether such a peak really occurs in a random SL or not depends on the details of the SL structure and will be discussed below. An important observation is that the derivation of Eq. (5) for the double-layer SL's is applicable also to the single-layer SL's. The intensity in the latters is obtained from (5) by putting $d_{1}^{A}=d_{2}^{B}=0$ or $d_{2}^{A}=d_{1}^{B}=0$.

The first model we shall consider more explicitly is the double-layer random SL in which the thicknesses of the first layers in both $A$ and $B$ blocks are the same, i.e., $d_{1}^{A}=d_{1}^{B}$. In this case $\alpha_{1}=\beta_{1}$ and $\gamma_{l}$ is independent of $l$. Hence, we put $\gamma_{l}=\gamma$ and also note that $\epsilon=\eta \xi$ (or $\xi=\eta^{*} \epsilon$ because $\left.|\eta|^{2}=1\right)$. Thus we find for the normalized intensity $\bar{I}=I_{D}$,

$$
I_{D}=|1-\eta|^{2} \frac{1-|\epsilon|^{2}}{|1-\epsilon|^{2}} .
$$

In the particularly simple case in which $A$ and $B$ occur with equal probabilities [i.e., $P(A)=P(B)=\frac{1}{2}$, where $P(A)$ and $P(B)$ are the probabilities for $A$ and $B$ blocks to occur in a sequence], Eq. (6) reduces to

$$
I_{D}=2(1-\cos \gamma) \frac{1-\cos ^{2} \Delta}{1-2 \cos \Phi \cos \Delta+\cos ^{2} \Delta},
$$

where $\Phi=(a+b) / 2$ and $\Delta=(a-b) / 2$. In Fig. 2(a), $I_{D}$ versus frequency is plotted for a (001)-AlAs/GaAs SL with $d_{1}^{A}=d_{1}^{B}=17 \AA$ (AlAs layers), $d_{2}^{A}=42 \AA$, and $d_{2}^{B}=20 \AA$ (GaAs layers). This choice of building blocks is the same as that used by Merlin et al. for a Fibonacci $\mathrm{SL}^{2}$ Here, we note that apart from the prefactor $2(1-\cos \gamma)$, Eq. (7) is identical to the $\mathrm{x}$-ray diffraction intensity derived by Hendricks and Teller for partially ordered layered systems based on a random sequence. ${ }^{8}$ The intensity has sharp peaks as well as small bumps. It also vanishes at frequencies satisfying the resonance conditions $\cos \gamma=1$ and $\cos \Delta= \pm 1$. The former equation is satisfied at frequencies $v=n \times 1.17 \mathrm{THz}$ ( $n$ is an integer) - at these frequencies the first layers become half-wave layers. The latter equations are satisfied at $v=n \times 0.757 \mathrm{THz}$.

The most striking feature in Fig. 2(a) is the sharp peak existing at $1.47 \mathrm{THz}$. This frequency [satisfying $\epsilon \simeq 1$ in Eq. (6)] is very close to 1.48 and $1.46 \mathrm{THz}$ for which the fifth- and third-order Bragg reflections occur in periodic SL's consisting of pure $A$ and pure $B$ blocks, respectively. We have verified that the TA phonon dispersion relations in the periodic SL's consisting of only $A$ blocks and that of only $B$ blocks have a common gap region on either side of $1.47 \mathrm{THz}$. Therefore, in these systems the Saxon-Hutner theorem ${ }^{9}$ is applicable at a narrow frequency range around $1.47 \mathrm{THz}$, that is, any frequency region which is a spectral gap for both a pure $A$-type SL and a pure $B$-type $\mathrm{SL}$ is necessarily a gap for any mixed SL of $A$ - and $B$-type blocks. This is the physical reason why we find a very narrow, sharp peak at this frequency.

From the ensemble-averaged intensity derived above, we can expect that even in random SL's definite structures should be observed in the phonon transmission (or reflection) spectra. In Fig. 2(b), the frequency dependence of the transmission rate calculated numerically is shown for the TA mode. This figure displays an average over the transmissions obtained from ten randomly generated sequences of $A$ and $B$ blocks used in Fig. 2(a) with $P(A)=P(B)=\frac{1}{2}$. The number of blocks used is equal to 100 in each sequence. As expected, the transmission rate reveals very distinctive structures which are in good agreement with the analytical result of Fig. 2(a). Transmission dips are quite significant at frequencies for which $I_{D}$ exhibits peaks, while the transmission is almost complete when $I_{D}$ vanishes. Small deviations from the perfect transmission at frequencies satisfying $I_{D}=0$ is $O\left(r_{21}^{2}\right)$. Note that the dip at $1.47 \mathrm{THz}$ is quite sharp with small fluctuations even for the small number of blocks (100) we use. 


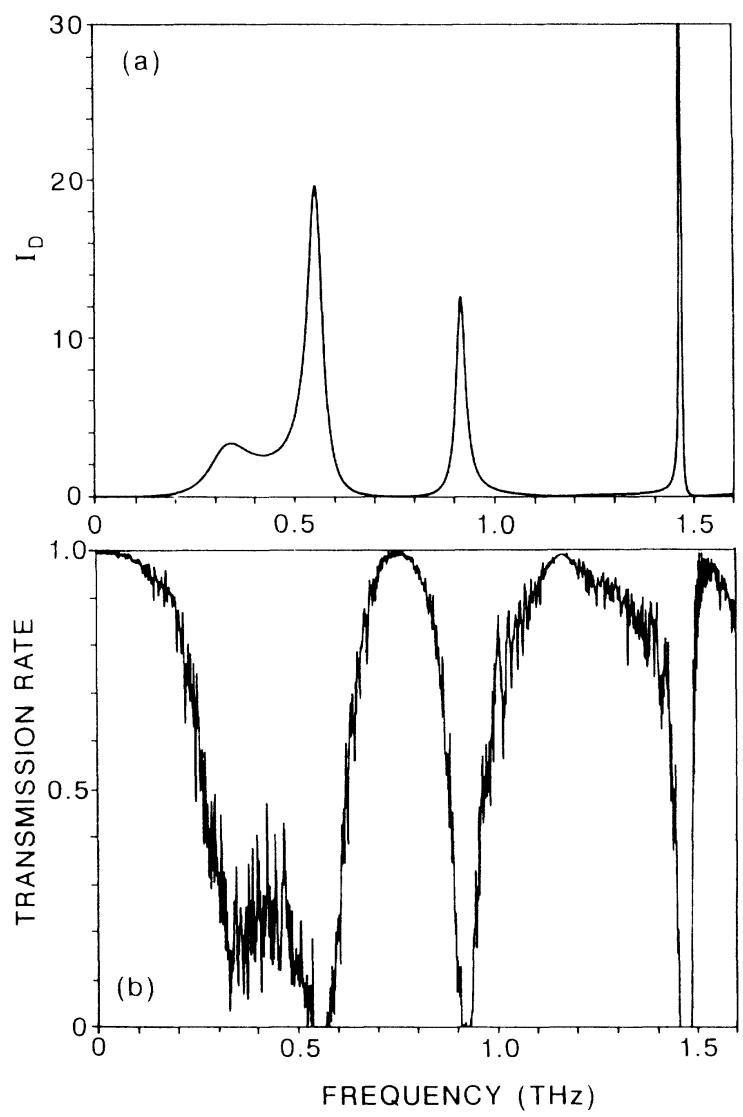

FIG. 2. (a) Intensity $I_{D}$ vs frequency in the double-layer random SL composed of (001)-oriented AlAs and GaAs layers (see the text). The phonon mode is TA. (b) Frequency dependence of the ensemble-averaged TA-phonon transmission in the double-layer SL, with the same parameters as in (a). The total number of blocks is one hundred and the results for ten independent random sequences of $A$ and $B$ blocks have been averaged.

The second model we shall consider is the single-layer random SL. As we have noted above, the expression for the intensity $\bar{I}=I_{S}$ is obtained, for instance, by putting $d_{2}^{A}=d_{1}^{B}=0, k_{1}=k_{A}, k_{2}=k_{B}, d_{1}^{A}=d_{A}$, and $d_{2}^{B}=d_{B}$ (see Fig. 1). In this case $1-\epsilon=(1-\eta)+(1-\xi)$ holds and we find from Eq. (5),

$$
\begin{aligned}
I_{S}=\frac{1}{|(1-\eta)+(1-\xi)|^{2}} & {\left[|1-\eta|^{2}\left(2-\xi-\xi^{*}\right)\right.} \\
& \left.+|1-\xi|^{2}\left(2-\eta-\eta^{*}\right)\right] .
\end{aligned}
$$

For a particularly simple case, with $P(A)=P(B)=\frac{1}{2}$, Eq. (8) reduces to

$$
I_{S}=\frac{(\cos \phi-\cos \delta)^{2}}{1-2 \cos \phi \cos \delta+\cos ^{2} \delta},
$$

where $\phi$ and $\delta$ are defined in terms of $\alpha=2 k_{A} d_{A}$ and $\beta=2 k_{B} d_{B}$ as $\phi=(\alpha+\beta) / 2$ and $\delta=(\alpha-\beta) / 2$. It is easily seen that $I_{S} \leq 1$.

Equation (9) is plotted in Fig. 3(a) against the phonon
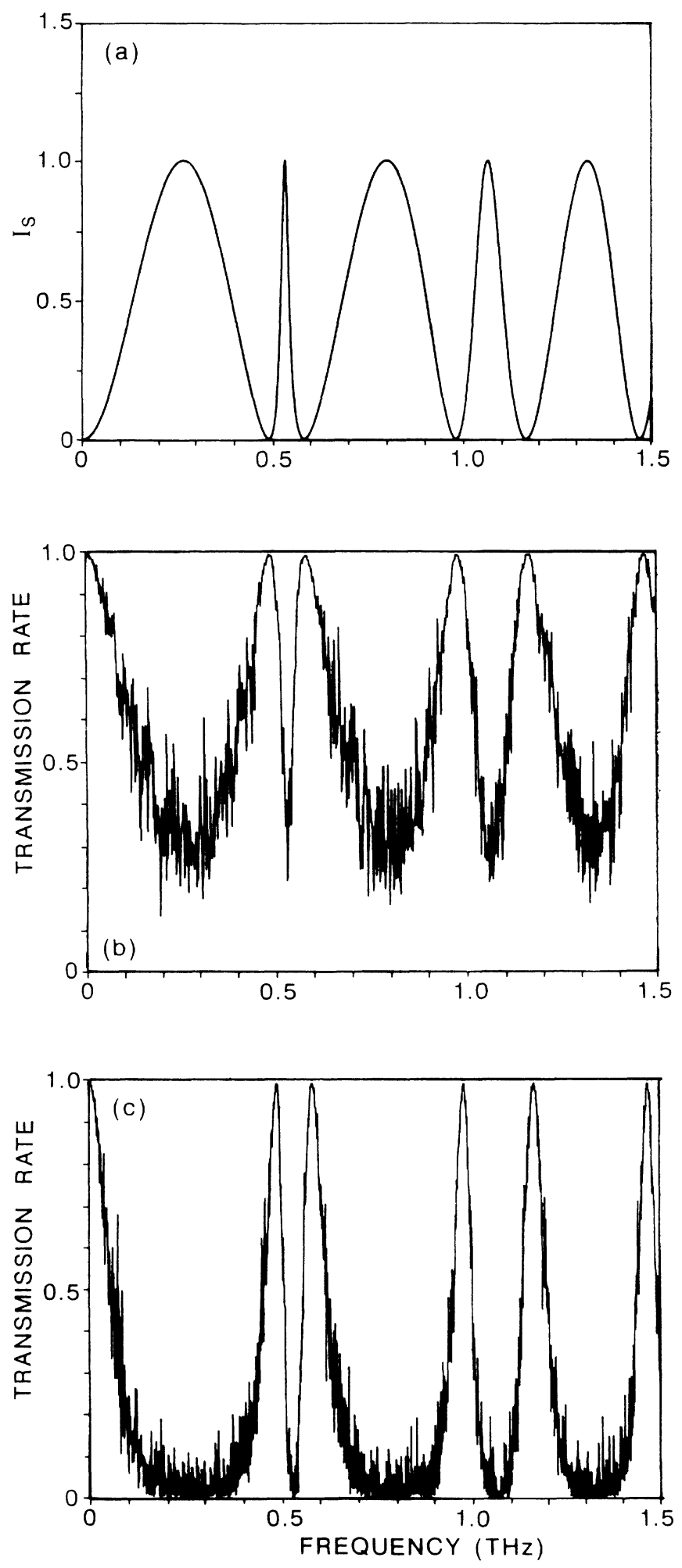

FIG. 3. (a) Intensity $I_{S}$ vs frequency in the single-layer random SL composed of $(001)$-oriented AlAs $(A)$ and GaAs $(B)$ layers with equal thickness, i.e., $34 \AA$. The phonon mode is TA. (b) Frequency dependence of the ensemble-averaged TAphonon transmission in the single-layer SL, with the same parameters as in (a). The total number of blocks is 200 and the results for ten independent random sequences of $A$ and $B$ blocks have been averaged. (c) Same as (b) but the total number of blocks in each sequence is 1000 . 
frequency of the TA mode for $d_{A}=d_{B}=34 \AA$. We find a regular oscillation of the ensemble averaged intensity, that is, the maxima $\left(I_{S}\right)_{\max }=1$ occur periodically at frequencies $v=n \times 0.266 \mathrm{THz}$ which are derived from $\cos ^{2} \phi=1$ and coincide with the ones at which the Bragg reflections occur in the periodic SL consisting of alternating $A$ and $B$ blocks. The minima $\left(I_{S}\right)_{\min }=0$ occur at $v=n \times 0.582$ $\mathrm{THz}$ and $v=n \times 0.492 \mathrm{THz}$. These frequencies are derived from the resonance condition $\cos \phi=\cos \delta$, or more explicitly, $\cos \alpha=1$ and $\cos \beta=1$, respectively. Note that for the Bragg reflection in periodic SL's, the normalized intensity $\bar{I}$ becomes infinity as $O(N)(N \rightarrow \infty)$, while in this random SL it is always $O\left(N^{0}\right)$ and bounded by unity. Accordingly, there exists no Bragg reflection in the single-layer random SL we consider.

In Fig. 3(b) we have displayed a numerical result for the ensemble-averaged transmission in the single-layer SL with the same parameters used for Fig. 3(a). Here, ten different random sequences of $200 A$ and $B$ blocks are generated with $P(A)=P(B)=\frac{1}{2}$. Besides the fluctuations of the transmission due to the finite size of the structure considered, the overall features are again in good agreement with the prediction of Fig. 3(a). Small deviations of the maximum transmission from unity are also $O\left(r_{21}^{2}\right)$. If we increase the number of blocks, the transmission dips become more significant and only those phonons satisfying the resonance condition can get through the SL's. This can be explicitly seen in Fig. 3(c), where the ensemble-averaged transmission of phonons through one thousand blocks is plotted. Thus, comparing with the periodic and nonperiodic (but based on a deterministic sequence) SL's studied hitherto, the single-layer random SL's have a quite effective filtering action on phonons over wide ranges of frequencies.

To conclude, we have investigated the transmission of acoustic phonons in randomly stacked multilayered systems consisting of two different building blocks. The calculation of the ensemble-averaged structure factor or the intensity of reflected phonons exhibits a very distinctive frequency dependence for both types of systems (double or single layered). Numerical calculations of the phonon transmission rate reveal quite well the characteristic features predicted for the infinite systems.

Here, we remark that the transmission rate calculated for any given random SL sample (without any ensemble averaging) shows large fluctuations. These fluctuations are, however, smeared out considerably if we average the transmission over a finite interval of frequency, and the result looks very similar to the ensemble-averaged one. The currently available phonon detectors do not possess the frequency resolution enough to resolve the fine structures in the predicted transmission. ${ }^{4}$ It is, therefore, quite probable that phonon spectroscopy and phonon imaging experiments that measure the transmission of high-frequency phonons in a random SL observe structures very similar to the ones we obtained by ensemble averaging.

In the present paper, we have considered SL's based on random sequences of blocks, i.e., without any correlations. An extension of this work is obtained by introducing correlations among $N$-consecutive blocks. For instance, the random version of the Fibonacci SL grown according to a three-state Markov process ${ }^{10}$ has already been studied by Merlin et al. ${ }^{3}$ using Raman scattering. The phonon spectra in the SL's based on the more general " $N$-state" Markov process is also interesting in regard to "speckles" 11 of phonons due to random fluctuations in a structure. These subjects are currently under consideration and will be discussed elsewhere.

One of us (S.T.) has benefited from conversations with J. P. Wolfe. The work at Hokkaido University was supported by a Grant-in-Aid for Scientific Research from the Ministry of Education, Science and Culture of Japan (Grant No. 0155001). F.N. acknowledges support from the Physics Department at the University of Michigan, the NSF through Grant No. PHY-82-17852, supplemented by funds from NASA, and Department of Energy Grant No. DE-84-ER45108.
*Permanent address: Physics Department, The University of Michigan, Ann Arbor, MI 48109-1120.

${ }^{1}$ For a recent review, see R. Merlin, IEEE J. Quantum Electron. QE-24, 1791 (1988).

${ }^{2}$ R. Merlin, K. Bajema, R. Clarke, F.-Y. Juang, and K. Bhatta. charya, Phys. Rev. Lett. 55, 1768 (1985).

${ }^{3}$ R. Merlin, K. Bajema, J. Nagle, and K. Ploog, J. Phys. (Paris) Colloq. 48, C5-503 (1987).

${ }^{4}$ V. Narayanamurti, H. L. Stormer, M. A. Chin, A. C. Gossard, and W. Wiegmann, Phys. Rev. Lett. 43, 2012 (1979); O. Koblinger, J. Mebert, E. Dittrich, S. Dottinger, W. Eisenmenger, P. V. Santos, and L. Ley, Phys. Rev. B 35, 9372 (1987); D. C. Hurley, S. Tamura, J. P. Wolfe, and H. Morkoc, Phys. Rev.
Lett. 58, 2446 (1987); S. Tamura, D. C. Hurley, and J. P. Wolfe, Phys. Rev. B 38, 1427 (1988).

${ }^{5}$ S. Tamura and J. P. Wolfe, Phys. Rev. B 36, 3491 (1987); D. C. Hurley, S. Tamura, J. P. Wolfe, K. Ploog, and J. Nagle, ibid. 37, 8829 (1988).

${ }^{6}$ S. Tamura and F. Nori, Phys. Rev. B 40, 9790 (1989).

${ }^{7}$ S. Tamura and J. P. Wolfe, Phys. Rev. B 38, 5610 (1988).

${ }^{8}$ S. Hendricks and E. Teller, J. Chem. Phys. 10, 147 (1942).

${ }^{9}$ See, for example, J. M. Ziman, Models of Disorder (Cambridge Univ. Press, London, 1979).

${ }^{10}$ M. R. Schroeder, Number Theory in Science and Communication (Springer-Verlag, Berlin, 1985), Chap. 30.

${ }^{11}$ A. Garg and D. Levine, Phys. Rev. Lett. 60, 2160 (1988). 
(a)

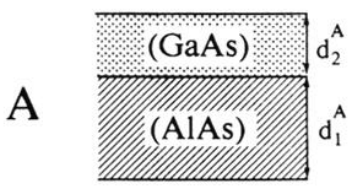

B

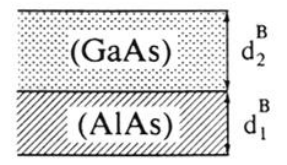

(b)

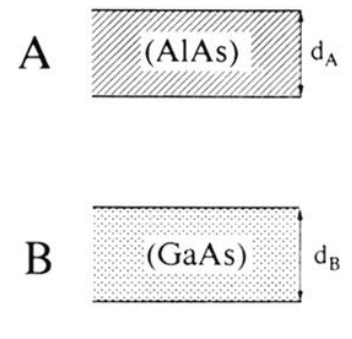

FIG. 1. Schematic representation of the building blocks $A$ and $B$ for (a) double-layer SL's and (b) single-layer SL's. AlAs and $\mathrm{GaAs}$ are assumed to be the constituents of layers in the numerical calculations. 